\title{
Wavy Leading Edge Airfoils Interacting with Anisotropic Turbulence
}

\author{
Fernando Gea-Aguilera, James Gill†, David Angland‡ \\ Faculty of Engineering and the Environment, \\ University of Southampton, Southampton, SO16 7QF, UK. \\ Xin Zhang, \\ The Hong Kong University of Science and Technology, \\ Clear Water Bay, Kowloon, Hong Kong SAR, China.
}

\begin{abstract}
Leading edge noise reductions caused by serrations have been shown to be sensitive to the length scales of vortical disturbances. In order to improve the understanding of wavy leading edge airfoils as a noise reduction technology, this paper examines the effects of anisotropy on turbulence-airfoil interaction noise by means of computational aeroacoutic simulations. A synthetic turbulence method is used to generate fully three-dimensional, divergence-free, homogeneous anisotropic turbulence, which is injected in a linearized Euler equation solver to model the noise generation. Moderate variations in turbulence length scales, which are representative of the anisotropy in aero-engine fan wakes, are tested for a NACA 0012 airfoil with a wavy leading edge. This work focuses on the noise sources in the near-field by examining the distortion of the turbulent structures and velocity spectra in the vicinity of the noise sources, the unsteady pressure and its spectral density on the airfoil surface, the magnitude-squared coherence between velocity and pressure fluctuations on the noise sources, and the correlation between noise sources along the span for various degrees of anisotropy. Numerical results show that small variations in the turbulence length scales can produce significant changes in the spectral content of the noise sources at the peak and root regions. The loudest noise source is always located in the root region for the cases examined and this source is mainly affected by the transverse velocity fluctuations. To reduce the correlation between noise sources in the peak and root regions, the ratio between the chordwise length scale and the amplitude of the serrations, and the ratio between the spanwise length scale and the wavelength of the leading edge should satisfy $l_{x} /\left(2 h_{w}\right)<1$ and $l_{z} / \lambda_{w} \leq 0.5$, respectively.
\end{abstract}

Keywords: computational aeroacoustics, anisotropic turbulence, wavy leading edge, broadband noise

\section{Introduction}

$\mathrm{T}$ HE aerospace industry is committed to reducing the environmental impact of aviation, including both air pollution and noise nuisance. For instance, stringent targets in terms of noise reduction have been set in Europe for 2050, which aim to reduce acoustic emissions by $65 \%$ compared to a baseline year of $2000 .{ }^{1}$ In the current generation of commercial aircraft, turbofan engines are a dominant source of broadband noise during take-off and approach. ${ }^{2}$ Broadband noise is mainly produced by the interaction of fan wakes with the Outlet Guide Vanes (OGVs), ${ }^{3}$ which is a source of leading edge noise. This source of noise is also relevant in Contra-Rotating Open Rotors (CRORs), as the front rotor wake impinges on the rear rotor blades, and in wind turbines.

*PhD Student, Airbus Noise Technology Centre.

${ }^{\dagger}$ Research Fellow, Airbus Noise Technology Centre.

$\ddagger$ Associate Professor, Airbus Noise Technology Centre, d.angland@soton.ac.uk.

$\S$ Swire Professor of Aerospace Engineering, Department of Mechanical and Aerospace Engineering, Associate Fellow, AIAA. 
Recent developments in aero-engines have focused on ultra-high bypass ratio turbofan engines for the coming generation of commercial aircraft. Such engines are characterized by a large bypass ratio, which may vary between 13 and 18, and a short nacelle, which reduces the separation between the fan and OGV stages and increases the importance of anisotropy in the fan wakes. ${ }^{4}$ In order to overcome the associated increase in the fan wake-OGV interaction noise, wavy leading edge airfoils have been proposed as a passive noise reduction technology over the past few years. ${ }^{5,6,7,8}$ Wavy leading edge airfoils are inspired by nature, as serrations in whale flippers and owl wings have been found to improve aerodynamic performance ${ }^{9,10,11,12}$ while reducing noise. ${ }^{5,6,8,13}$ Figure 1 shows a schematic of the locations where anisotropic turbulence contributes to the noise generation in a turbofan engine, and an OGV with wavy leading edge.

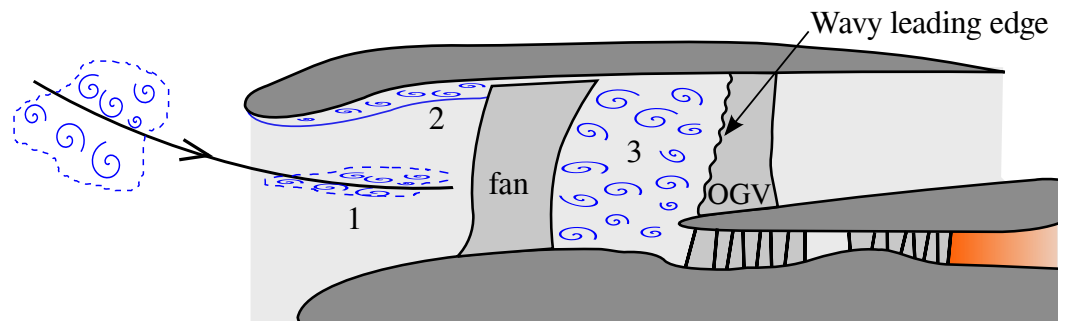

Figure 1. Anisotropic turbulence in a turbofan engine with wavy leading edge on the OGVs. (1) Turbulence ingestion, (2) boundary layer, and (3) fan wakes.

Recent experimental, numerical, and analytical studies ${ }^{6,8,14,15}$ highlighted the importance of the length scales of the vortical disturbances or turbulence as a key parameter in the noise reduction of wavy leading edge airfoils. However, the majority of previous works were based on the flat plate assumption ${ }^{8,16}$ and used simplified representations for the turbulence, such as harmonic gusts ${ }^{14}$ or isotropic turbulence. ${ }^{8}$

Unlike previous works that focused on flat plates and isotropic turbulence, the present work studies the noise generation mechanisms of an airfoil with wavy leading edge in the presence of anisotropic turbulence. Although the effects of anisotropic turbulence have been shown to be limited in airfoils with straight leading edge, ${ }^{17}$ wavy leading edge airfoils are expected to be more sensitive to anisotropic turbulence due to additional three-dimensional mechanisms caused by the amplitude and wavelength of the leading edge serrations. In this work, Computational AeroAcoustic (CAA) simulations are performed by means of a synthetic turbulence method to generate fully three-dimensional anisotropic turbulence and a linearized Euler equation (LEE) solver for the noise generation. The following topics are addressed here to examine the noise generation mechanisms in the near-field:

- The distortion of the turbulent structures and velocity spectra in the vicinity of the noise sources.

- The unsteady pressure and its spectral density on the airfoil surface.

- The influence of the distorted velocity fluctuations on the noise source through computation of the magnitude-squared coherence between velocity and pressure fluctuations.

- The correlation between noise sources along the span for various degrees of anisotropy.

\section{Previous Work}

Early works in the field focused on the aerodynamic advantages of wavy leading edge airfoils. For instance, Soderman ${ }^{9}$ reported a shift in the maximum lift coefficient towards higher incidences along with a drag reduction when using small and sharp serrations. Similar results were found by Miklosovic et al. ${ }^{10}$ and Van Nierop et al. ${ }^{11}$ in wind tunnel experiments and an analytical model of airfoils based on a humpback whale flipper with large tubercles on the leading edge. Using Large Eddy Simulations (LES), Skillen et al. ${ }^{12}$ showed that this aerodynamic improvement at high incidence was related to secondary flows induced by spanwise pressure gradients in the leading edge region. This caused the boundary layer to become turbulent in the vicinity of the airfoil leading edge, which delayed the flow separation.

Additionally to the enhanced aerodynamic performance, airfoils with wavy leading edge have also been reported to reduce noise. Hersh et al. ${ }^{5}$ performed a set of experiments on stationary and rotating NACA 0012 airfoils with sharp serrations, and showed a significant reduction in tonal noise (approximately 4 to $8 \mathrm{~dB}$ ) caused by wake vortex shedding at high incidence. Sinusoidal profiles were tested by Narayanan 
et $a l .{ }^{16}$ on flat plates, and concluded that leading edge serrations are more sensitive to the amplitude of the undulations than the wavelength. This finding was also reported by Chaitanya et al. 6,7 in an extensive experimental study that identified an optimum geometry for the undulations. The maximum noise reduction was observed for cases with the turbulence integral length scale being half of the serration wavelength. Recently, the acoustic properties of innovative leading edge geometries, such as dual-frequency, chopped-peak and slitted-root serrated airfoils, have also been investigated experimentally to maximize the noise reduction. ${ }^{18,19}$

Analytical models have also been developed to obtain noise predictions from wavy leading edge airfoils. For instance, Roger et al. ${ }^{20}$ extended Amiet's model ${ }^{21}$ by means of strip theory to account for leading edge undulations, which were characterized as a periodically changing sweep angle. The study examined the supercritical and subcritical behaviour of the vortical gusts as a key parameter in the noise reduction mechanism. Alternatively, Mathews and Peak ${ }^{15}$ derived the Green's function for a flat plate with wavy leading edge radiating to the far field. The interaction between a number of eddies and the flat plate showed the difficulty in finding an optimum geometry to reduce the noise caused by eddies with different parameters to define its size, amplitude and spatial location.

Several numerical studies have investigated the effects of wavy leading edges on noise. In CAA solvers, the interaction of vortical disturbances with a wavy leading edge airfoil is typically modeled by means of the full- or linearized Euler equations. For instance, Lau et al. ${ }^{14}$ focused on the interaction between a harmonic gust and a flat plate with wavy leading edge. Numerical results showed that the noise reduction increases when the ratio of the leading edge amplitude to the gust wavelength is increased. This result suggests that the size of the vortical structures in comparison with the geometry of the wavy leading edge might be an important parameter in the noise reduction. Clair et al. ${ }^{22}$ performed a numerical study on a NACA 65(12)-10 airfoil with an undulating leading edge. In this case, synthetic turbulence based on a summation of Fourier modes was used to model the transverse velocity component, which is perpendicular to the airfoil chord. The comparison with experimental results confirmed the noise reduction in the wavy leading edge airfoil. However, significant discrepancies were reported between numerical an experimental noise levels at high frequencies, which were attributed to the absence of oblique gusts in the vortical disturbances. A more realistic description of the turbulent flow was used by Kim et al. ${ }^{8}$ to study the noise reduction mechanism in a number of flat plates with various amplitudes of the wavy leading edge. By using a synthetic eddy method to realize fully three-dimensional isotropic turbulence, Kim et al. ${ }^{8}$ found that the size of the eddies relative to the amplitude of the wavy leading edge plays a significant role in the noise reduction.

\section{CAA method}

The present work uses a high-order finite difference numerical solver that has been used in prior CAA studies of turbulence-airfoil/cascade interaction noise. ${ }^{17,23,24,25}$ In the current investigation, the LEEs are solved in the time-domain by using a 4th-order compact spatial scheme. ${ }^{26}$ The numerical solution is filtered after each time-step for numerical stability. ${ }^{27}$ The time derivatives are obtained by using a 4-6 stage Low Dispersion and Dissipation Runge-Kutta (LDDRK) scheme. ${ }^{28}$ A slip-wall boundary condition is used on the airfoil surface. Additionally, implicit buffer zones $^{29}$ are applied in order to prevent sound waves from being reflected at the edge of the CAA domain.

Numerical simulations presented in this work were run with mean flow density $\rho_{\infty}=1.2 \mathrm{~kg} / \mathrm{m}^{3}$ and speed of sound $c_{\infty}=340 \mathrm{~m} / \mathrm{s}$. Turbulence spectra are normalized by using $U_{r e f}=1 \mathrm{~m} / \mathrm{s}$. The reference value for the Sound Pressure Level (SPL) is $2 \times 10^{-5} \mathrm{~N} / \mathrm{m}^{2}$.

All computations used the IRIDIS high performance computing facility at the University of Southampton.

\section{Anisotropic Synthetic Turbulence}

In this section, a synthetic turbulence method based on digital filters is presented to generate fully threedimensional homogeneous anisotropic turbulence ${ }^{\mathrm{a}}$. The resulting fluctuating velocity flow is obtained from the contribution of $P$ small particles or eddies that define the whole source region where the turbulence

\footnotetext{
${ }^{a}$ In homogeneous turbulence, one- and two-point statistics are invariant under axis translations, such as shifts in the origin of the reference frame. Therefore, the root-mean-square of the velocity fluctuations and turbulence length scales are constant in any spatial direction in which the turbulent flow is homogeneous. Unlike isotropic turbulence, anisotropic turbulence may present different root-mean-square velocity fluctuations and turbulence length scales in each spatial direction.
} 
is prescribed. Each eddy introduces a fluctuating velocity field, $\mathbf{u}_{p}^{\prime}=\left(u_{x, p}^{\prime}, u_{y, p}^{\prime}, u_{z, p}^{\prime}\right)$, around its center, $\left(x_{p}, y_{p}, z_{p}\right)$, that vanishes far from it, thus acting as a spatial filter.

Anisotropic Gaussian eddies are injected locally into the CAA domain through an injection plane, as shown in Figure 2(a). To this end, the governing equations of three-dimensional Gaussian eddies that were presented by Gea-Aguilera et al. ${ }^{23}$ to generate isotropic turbulence have been extended to account for anisotropy. This was realized by changing the size of the eddies in different spatial directions, i.e., by modifying the length of the spatial filters. Thus, the fluctuating velocity field introduced by the $p^{\text {th }}$ three-dimensional anisotropic Gaussian eddy is given by,

$$
\begin{aligned}
& u_{x, p}^{\prime}(\mathbf{x})=\frac{u_{0}^{\prime} \sqrt{\pi \Delta_{e, x} \Delta_{e, y} \Delta_{e, z}}}{\left(l_{x} l_{y} l_{z}\right)^{1 / 6}}\left[\frac{\epsilon_{2, p}\left(z-z_{p}\right)}{l_{z}^{2}}-\frac{\epsilon_{3, p}\left(y-y_{p}\right)}{l_{y}^{2}}\right] A_{p}, \\
& u_{y, p}^{\prime}(\mathbf{x})=\frac{u_{0}^{\prime} \sqrt{\pi \Delta_{e, x} \Delta_{e, y} \Delta_{e, z}}}{\left(l_{x} l_{y} l_{z}\right)^{1 / 6}}\left[\frac{\epsilon_{3, p}\left(x-x_{p}\right)}{l_{x}^{2}}-\frac{\epsilon_{1, p}\left(z-z_{p}\right)}{l_{z}^{2}}\right] A_{p}, \\
& u_{z, p}^{\prime}(\mathbf{x})=\frac{u_{0}^{\prime} \sqrt{\pi \Delta_{e, x} \Delta_{e, y} \Delta_{e, z}}}{\left(l_{x} l_{y} l_{z}\right)^{1 / 6}}\left[\frac{\epsilon_{1, p}\left(y-y_{p}\right)}{l_{y}^{2}}-\frac{\epsilon_{2, p}\left(x-x_{p}\right)}{l_{x}^{2}}\right] A_{p},
\end{aligned}
$$

where $\epsilon_{i, p}$ can randomly take \pm 1 for $i=1,2,3, u_{0}^{\prime}$ is a characteristic velocity term that controls the amplitude of the anisotropic eddies, $\Delta_{e, x}, \Delta_{e, y}$ and $\Delta_{e, z}$ correspond to the separation between eddy centers in the $x$, $y$ and $z$ directions, respectively, and $A_{p}$ corresponds to the exponential term,

$$
A_{p}=\exp \left[-\frac{\pi\left(x-x_{p}\right)^{2}}{2 l_{x}^{2}}\right] \exp \left[-\frac{\pi\left(y-y_{p}\right)^{2}}{2 l_{y}^{2}}\right] \exp \left[-\frac{\pi\left(z-z_{p}\right)^{2}}{2 l_{z}^{2}}\right] .
$$

It should be noted that each eddy introduces a fluctuating velocity field that is divergence-free, i.e. $\nabla \cdot \mathbf{u}_{p}^{\prime}=0$, which allows the local injection of synthetic eddies without generating spurious noise sources in the CAA domain.

In this work, the integral length scale of a turbulent flow is defined as,

$$
l_{i j}^{(k)}=\int_{0}^{\infty} \frac{\left\langle u_{i}^{\prime}\left(\mathbf{x}+r \mathbf{e}_{k}\right) u_{j}^{\prime}(\mathbf{x})\right\rangle}{\left\langle u_{i}^{\prime}(\mathbf{x}) u_{j}^{\prime}(\mathbf{x})\right\rangle} \mathrm{d} r
$$

where $u_{i}^{\prime}$ represents the $i^{\text {th }}$ fluctuating velocity component, $r$ is the separation between two points in the $k^{\text {th }}$ direction, $\mathbf{e}_{k}$ is a unit vector in the $k^{\text {th }}$ direction, and $\langle$.$\rangle is the ensemble average operator. Therefore, l_{i j}^{(k)}$ is the integral length scale of the $i^{\text {th }}$ and $j^{\text {th }}$ fluctuating velocity components in the $k^{\text {th }}$ spatial direction. For isotropic turbulence, $l_{11}^{(1)} / l_{22}^{(1)}=l_{11}^{(1)} / l_{33}^{(1)}=2$ and $l_{11}^{(1)}=l_{22}^{(2)}=l_{33}^{(3)}$. To simplify the notation, the turbulence length scales $l_{x}, l_{y}$ and $l_{z}$ in Equations 1 to 3, correspond to $l_{11}^{(1)}, l_{22}^{(2)}$ and $l_{33}^{(3)}$, respectively, as defined in Equation 5. These turbulence length scales are representative of the size of the eddies in different spatial directions.

The resulting velocity spectra that are obtained by using three-dimensional anisotropic Gaussian eddies are given by,

$$
\begin{aligned}
& \Phi_{11}(\mathbf{k})=\frac{u_{0}^{\prime 2}}{\pi^{4}}\left(l_{x} l_{y} l_{z}\right)^{5 / 3}\left(k_{y}^{2}+k_{z}^{2}\right) \exp \left(-\frac{l_{x}^{2} k_{x}^{2}+l_{y}^{2} k_{y}^{2}+l_{z}^{2} k_{z}^{2}}{\pi}\right), \\
& \Phi_{22}(\mathbf{k})=\frac{u_{0}^{\prime 2}}{\pi^{4}}\left(l_{x} l_{y} l_{z}\right)^{5 / 3}\left(k_{x}^{2}+k_{z}^{2}\right) \exp \left(-\frac{l_{x}^{2} k_{x}^{2}+l_{y}^{2} k_{y}^{2}+l_{z}^{2} k_{z}^{2}}{\pi}\right), \\
& \Phi_{33}(\mathbf{k})=\frac{u_{0}^{\prime 2}}{\pi^{4}}\left(l_{x} l_{y} l_{z}\right)^{5 / 3}\left(k_{x}^{2}+k_{y}^{2}\right) \exp \left(-\frac{l_{x}^{2} k_{x}^{2}+l_{y}^{2} k_{y}^{2}+l_{z}^{2} k_{z}^{2}}{\pi}\right),
\end{aligned}
$$

where $\mathbf{k}=\left(k_{x}, k_{y}, k_{z}\right)$ is the wave-vector and the vortical wavenumber components. The turbulent kinetic energy is defined as,

$$
K=\frac{1}{2} \iiint_{-\infty}^{+\infty}\left[\Phi_{11}(\mathbf{k})+\Phi_{22}(\mathbf{k})+\Phi_{33}(\mathbf{k})\right] \mathrm{d} \mathbf{k}=\frac{1}{2}\left(u_{x, r m s}^{\prime 2}+u_{y, r m s}^{\prime 2}+u_{z, r m s}^{\prime 2}\right),
$$


where the mean-square of the velocity fluctuations are,

$$
u_{x, r m s}^{\prime 2}=\frac{u_{0}^{\prime 2}}{2} \frac{l_{x}^{2 / 3}}{l_{y}^{4 / 3} l_{z}^{4 / 3}}\left(l_{y}^{2}+l_{z}^{2}\right), \quad u_{y, r m s}^{\prime 2}=\frac{u_{0}^{\prime 2}}{2} \frac{l_{y}^{2 / 3}}{l_{x}^{4 / 3} l_{z}^{4 / 3}}\left(l_{x}^{2}+l_{z}^{2}\right), \quad u_{z, r m s}^{\prime 2}=\frac{u_{0}^{\prime 2}}{2} \frac{l_{z}^{2 / 3}}{l_{x}^{4 / 3} l_{y}^{4 / 3}}\left(l_{x}^{2}+l_{y}^{2}\right)
$$

Following the study of Gea-Aguilera et al. ${ }^{23}$ for isotropic turbulence, the correct turbulence statistics can be recovered provided that the radius of the eddies satisfies $r_{e, x} \geq 3 l_{x} / 2, r_{e, y} \geq 3 l_{y} / 2$ and $r_{e, z} \geq 3 l_{z} / 2$, and the eddy spacing in each spatial direction is $\Delta_{e, x} \leq l_{x} / 2, \Delta_{e, y} \leq l_{y} / 2$ and $\Delta_{e, z} \leq l_{z} / 2$. The statistics of the synthetic turbulence are validated in a three-dimensional CAA domain with uniform grid spacing of $1.1 \times 10^{-3} \mathrm{~m}$. The freestream Mach number was set to $M_{\infty}=0.3$ and the turbulence inflow parameters are $u_{0}^{\prime 2}=3 \mathrm{~m}^{2} / \mathrm{s}^{2}, l_{x}=0.008 \mathrm{~m}, l_{y}=2 l_{x}$, and $l_{z}=3 l_{x}$. One-dimensional spectra of three-dimensional turbulence,

$$
E_{i j}(f)=\frac{4 \pi}{U_{x}} \int_{-\infty}^{+\infty} \int_{-\infty}^{+\infty} \Phi_{i j}\left(k_{x}, k_{y}, k_{z}\right) \mathrm{d} k_{y} \mathrm{~d} k_{z}
$$

are computed from fluctuating velocity samples and compared to analytical expressions in Figure 2(b). It should be noted that the one-dimensional spectrum $E_{i j}(f)$ corresponds to the Power Spectral Density (PSD) of the velocity fluctuations in the $i^{\text {th }}$ and $j^{\text {th }}$ directions. The multitaper spectral analysis of Thomson ${ }^{30}$ has been used to calculate the numerical spectral density of the fluctuating velocity. Results show the effectiveness of the current anisotropic synthetic turbulence method to accurately reproduce homogeneous anisotropic synthetic turbulence with Gaussian spectra.

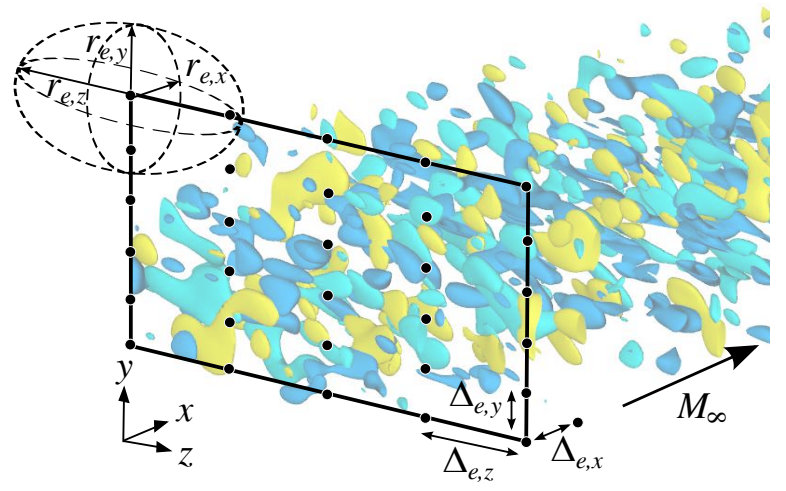

(a) Numerical implementation of turbulence injection plane.

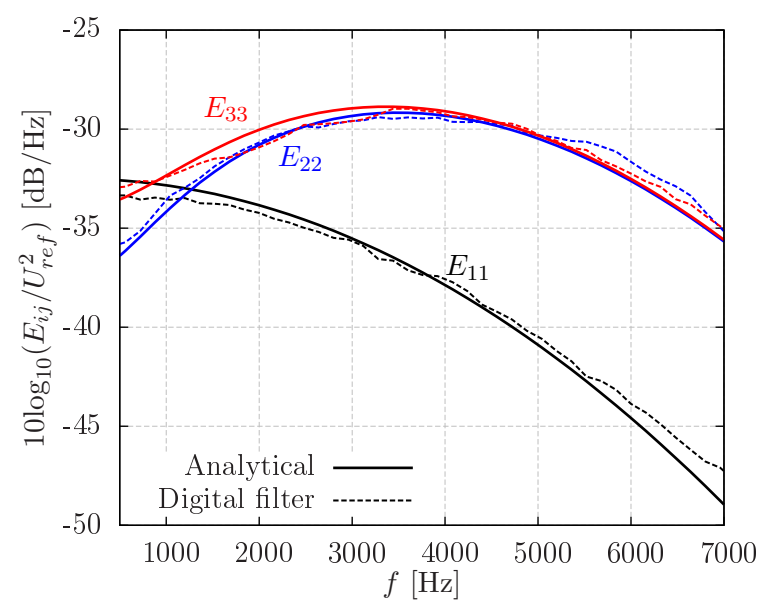

(b) One-dimensional spectra of anisotropic Gaussian turbulence with $u_{0}^{\prime 2}=3 \mathrm{~m}^{2} / \mathrm{s}^{2}, l_{x}=0.008 \mathrm{~m}, l_{y}=2 l_{x}$ and $l_{z}=3 l_{x}$.

Figure 2. Three-dimensional anisotropic turbulence.

\section{Wavy Leading Edge Geometry and Computational Setup}

The parameters that define the geometry of the wavy leading edge airfoil are shown in Figure 3, where $2 h_{w}$ is the amplitude of the undulations, $\lambda_{w}$ is the wavelength of the leading edge, and $c_{0}$ is the airfoil mean chord. In this work, the effects of anisotropy are tested on a NACA 0012 airfoil at zero angle of attack with $h_{w} / c_{0}=0.1$ and $\lambda_{w} / c_{0}=0.10 \dot{6}$, where $c_{0}=0.15 \mathrm{~m}$. This combination of parameters was found to produce a maximum noise reduction on flat plates when interacting with isotropic turbulence with the integral length scale set to $l_{11}^{(1)}=2 l_{22}^{(1)}=\lambda_{w} / 2{ }^{6,7}$ The profile of the NACA 0012 airfoil with wavy leading edge at each spanwise location, $z$, is defined by Chaitanya et al. ${ }^{6,7}$ as,

$$
y(x, z)= \begin{cases}f\left(x / c_{0}\right), & \text { if } 0 \leq x / c_{0}<2 / 3, \\ f(x / c(z)), & \text { if } 2 / 3 \leq x / c(z) \leq 1,\end{cases}
$$

where $c(z)=c_{0}+h_{w} \sin \left(2 \pi z / \lambda_{w}\right)$ is the local airfoil chord and $x / c_{0}=0$ corresponds to the trailing edge. 


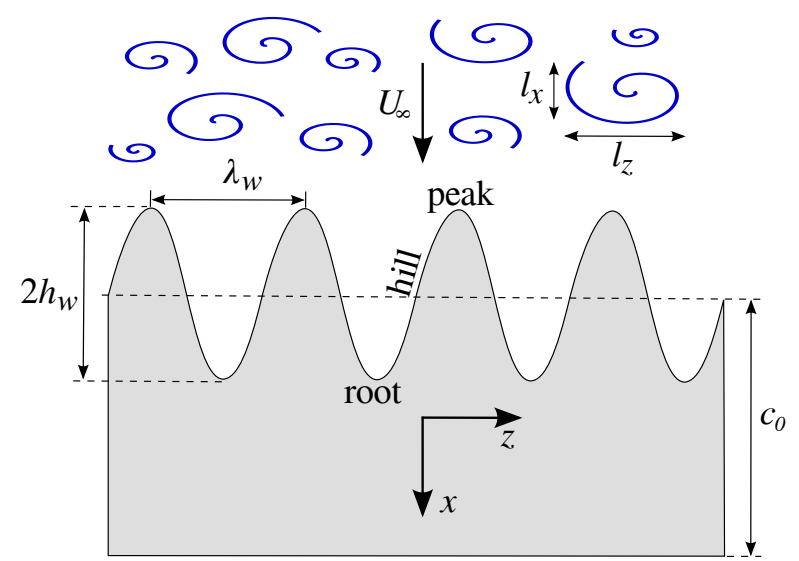

(a) Schematic of the wavy leading edge airfoil and turbulence length scales.

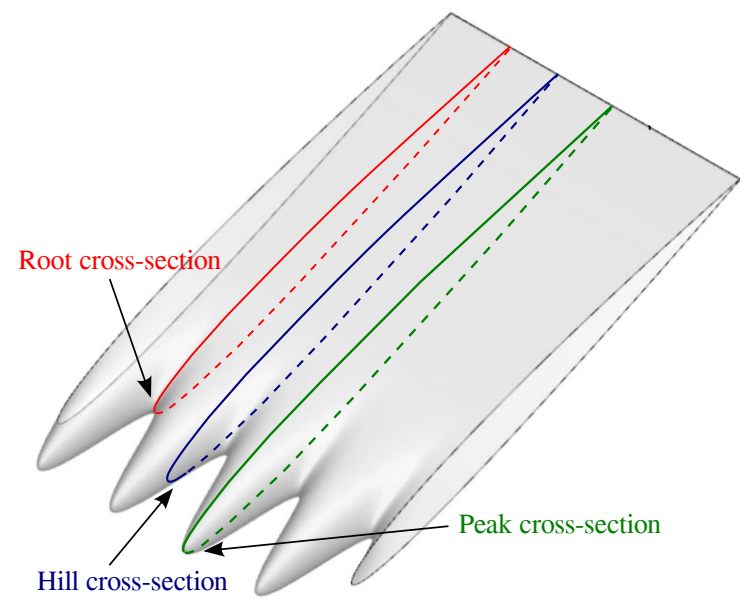

(b) Root, hill and peak cross-sections of a NACA 0012 airfoil with wavy leading edge.

Figure 3. Parameters and definitions for a wavy leading edge airfoil interacting with anisotropic turbulence.

Moderate variations in the turbulence length scales, which are representative of the anisotropy in fan wakes, are tested here for the chosen airfoil configuration. The purpose is to study the effects of $l_{x} /\left(2 h_{w}\right)$ and $l_{z} / \lambda_{w}$ on the noise generation mechanism of airfoils with wavy leading edge. Optimizing the geometry of the undulations based on the different length scales in the fan wakes would be useful for aero-engine manufacturers to adapt their OGV designs. Table 1 shows the configurations that are investigated in the current work. For all the configurations, $u_{0}^{\prime 2}=3 \mathrm{~m}^{2} / \mathrm{s}^{2}$, which corresponds to a turbulence intensity of $1.7 \%$ in the case of isotropic turbulence $\left(l_{x}=l_{y}=l_{z}\right)$.

The CAA simulations were performed in a computational domain that extends to approximately $4 c$ in all directions from the airfoil center. The grid resolution is at least 8 Points-Per-Wavelength (PPW) for the smallest vortical waves that are propagated without significant dispersion and dissipation errors by the CAA code. It has been ensured that the mesh is able to perform accurate noise predictions up to a frequency of $5 \mathrm{kHz}$ for a freestream Mach number of $M_{\infty}=0.3$ (chord-based reduced frequency $f c_{0} / U_{\infty} \approx 7.35$ ), which covers the frequency range in which leading edge noise is the dominant source of noise. This is because airfoil self-noise, which is not included in the CAA simulations presented in the present work, is expected to be the dominant source of noise at relatively high frequencies. For instance, Chaitanya et $_{\text {al. }}{ }^{31}$ found that the self-noise of a NACA 0012 airfoil with straight leading edge was dominant for frequencies above $f c_{0} / U_{\infty} \approx 8$ in their experiment.

In previous CAA studies of wavy leading edge airfoils, the width of the CAA domain was restricted to $\lambda_{w}{ }^{14,22}$ or $2 \lambda_{w}{ }^{8}$ to reduce the computational expense with approximately 13 grid points per $\lambda_{w}{ }^{8}$ to define the undulations in the spanwise direction. In contrast, the width of the CAA simulations in this work extends to $4 \lambda_{w}$ in order to allow the injection of synthetic eddies stretched in the spanwise direction without truncation. This ensures that the correlation between velocity fluctuations on opposite spanwise edges of the CAA domain is zero. Furthermore, 52 grid points per $\lambda_{w}$ have been used here to generate a well-defined and smooth geometry at the leading edge, while ensuring the correct spatial resolution in the spanwise direction due to variations in the length scales of the eddies. The total number of grid points in the CAA domain is approximately $45.7 \times 10^{6}$. Periodic boundary conditions are set in the spanwise direction, as used in previous CAA studies of three-dimensional airfoils using isotropic turbulence. ${ }^{8,22}$

It is well known that simulations made using uniform mean flows over-predict leading edge noise from thick airfoils at high frequencies, and that viscosity can be neglected without significant loss of accuracy in the noise predictions. ${ }^{22,32}$ Consequently, an inviscid mean flow is used in the CAA simulations to account for mean flow gradients around the NACA 0012 airfoil. This allows the turbulent structures to be distorted in the vicinity of the airfoil leading edge, which is essential for correct leading edge noise predictions of thick airfoils. ${ }^{32}$ The present study also examines the distortion of the velocity spectra in the vicinity of the airfoil with wavy leading edge, as this has been highlighted as a key factor in turbulence-airfoil interaction noise. $^{23,33,34}$ 


\begin{tabular}{|c|c|c|c|c|c|c|c|}
\hline$l_{x}[\mathrm{~m}]$ & $l_{z}[\mathrm{~m}]$ & $l_{x} /\left(2 h_{w}\right)$ & $l_{z} / \lambda_{w}$ & $u_{x, r m s}^{\prime}[\mathrm{m} / \mathrm{s}]$ & $u_{y, r m s}^{\prime}[\mathrm{m} / \mathrm{s}]$ & $u_{z, r m s}^{\prime}[\mathrm{m} / \mathrm{s}]$ & $K\left[\mathrm{~m}^{2} / \mathrm{s}^{2}\right]$ \\
\hline \hline 0.004 & 0.008 & $0.1 \dot{3}$ & 0.50 & 2.38 & 3.76 & 3.76 & 17.01 \\
\hline 0.016 & 0.008 & $0.5 \dot{3}$ & 0.50 & 3.78 & 2.99 & 2.99 & 16.07 \\
\hline 0.032 & 0.008 & $1.0 \dot{6}$ & 0.50 & 4.76 & 3.47 & 3.47 & 23.39 \\
\hline \hline 0.008 & 0.008 & $0.2 \dot{6}$ & 0.50 & 3.00 & 3.00 & 3.00 & 13.50 \\
\hline \hline 0.008 & 0.004 & $0.2 \dot{6}$ & 0.25 & 3.76 & 3.76 & 2.38 & 17.01 \\
\hline 0.008 & 0.012 & $0.2 \dot{6}$ & 0.75 & 2.92 & 2.92 & 3.43 & 14.41 \\
\hline 0.008 & 0.016 & $0.2 \dot{6}$ & 1.00 & 2.99 & 2.99 & 3.78 & 16.07 \\
\hline
\end{tabular}

Table 1. Length scales of anisotropic turbulence to study the effect of $l_{x} / h_{w}$ and $l_{z} / \lambda_{w}$ in the noise generation mechanism of airfoils with wavy leading edge. All cases have $u_{0}^{\prime 2}=3 \mathrm{~m}^{2} / \mathrm{s}^{2}$ and $l_{y}=0.008 \mathrm{~m}$. The geometry of the undulations is defined by $h_{w}=0.015 \mathrm{~m}$ and $\lambda_{w}=0.016 \mathrm{~m}$.

\section{Wavy Leading Edge Airfoils Interacting with Anisotropic Turbulence}

In this section, CAA simulations of a NACA 0012 airfoil with a wavy leading edge are presented using fully three-dimensional anisotropic Gaussian turbulence. It should be noted that synthetic turbulence with Gaussian spectra provides a first approximation to the spectral content in a turbulent flow, which is sufficient to study the effects of anisotropic turbulence on leading edge noise from airfoils with serrations. However, Gaussian spectra cannot account for the correct energy decay at high frequencies, and therefore, they are not suitable to accurately reproduce the anisotropy in realistic turbulent flows.

The noise generation mechanism is investigated in the near-field to improve understanding of the effects of anisotropic turbulence on leading edge noise. To provide a physical insight, the distortions of the turbulence spectra in the leading edge region are investigated, and their contribution to the noise sources on the airfoil surface is examined by means of the magnitude-squared coherence between pressure and velocity fluctuations. The spectral density of the pressure fluctuations on the airfoil surface is studied at peak, hill and root (or valley) cross-sections for various degrees of anisotropy in the oncoming turbulent flow. The spanwise correlation between the pressure fluctuations at the noise sources are also investigated. Proposed values for $l_{x} /\left(2 h_{w}\right)$ and $l_{z} / \lambda_{w}$ are given to decrease the correlation between noise sources for wavy leading edge airfoils in realistic turbulent flows, and therefore, to optimize the geometry of the serrations to reduce leading edge noise when anisotropy is important.

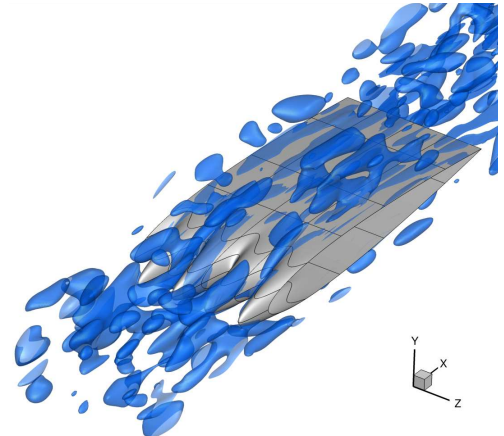

(a) Turbulent structures stretched in the chordwise direction $\left(l_{x}=0.016 \mathrm{~m}\right.$, $\left.l_{y}=l_{z}=0.008 \mathrm{~m}\right)$.

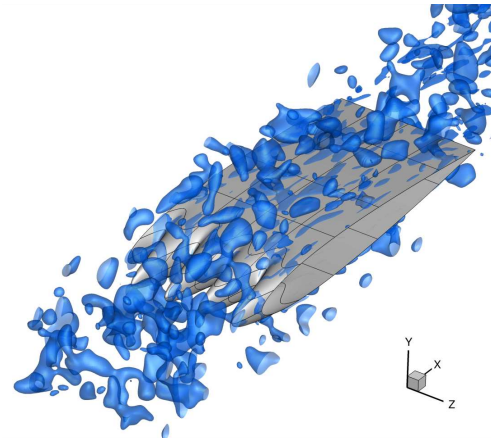

(b) Isotropic turbulence $\left(l_{x}=l_{y}=l_{z}=\right.$ $0.008 \mathrm{~m})$.

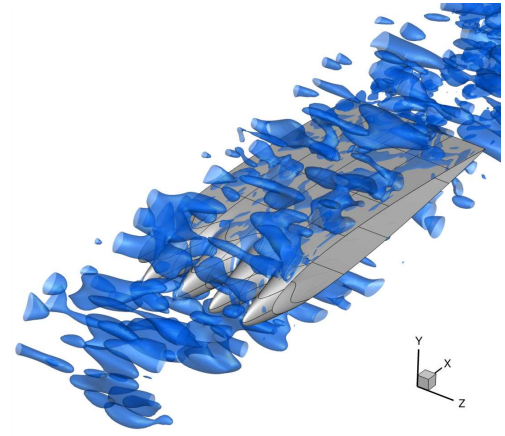

(c) Turbulent structures stretched in the spanwise direction $\left(l_{x}=l_{y}=0.008 \mathrm{~m}\right.$, $\left.l_{z}=0.016 \mathrm{~m}\right)$.

Figure 4. Iso-contours of instantaneous fluctuating velocity magnitude.

\section{VI.A. Distortion of Turbulent Structures and Velocity Spectra}

The distortion of the turbulent structures in the leading edge region is believed to be responsible for the noise reduction of thick airfoils at high frequencies. Previous works examined the distortion of harmonic gusts $^{32,35}$ and isotropic turbulence ${ }^{23,34}$ in two-dimensional CAA simulations of isolated airfoils. Additionally, experiments of airfoils with straight leading edge have also been performed to study the turbulent flow 
features in the leading edge region. ${ }^{33,31}$ This section provides the spectral analysis of streamwise, transverse and spanwise velocity fluctuations in the vicinity of a thick airfoil with a wavy leading edge.

Figure 5 shows the injected turbulence spectra (represented by the subscript $\infty$ ) and the distorted spectra in the vicinity of peak and root cross-sections assuming isotropic turbulence $\left(l_{x}=l_{y}=l_{z}\right)$. It should be noted that the injected transverse and spanwise turbulence spectra collapse for isotropic turbulence. Overall, larger distortions of the turbulence spectra can be found in the root region than in the peak region. This is consistent with the findings of Skillen et al., ${ }^{12}$ who reported a strong spanwise pressure gradient along the leading edge that forces the streamlines to deflect from the peak to the root regions, and consequently, the generation of a secondary flow towards the root region.

In the peak region (see Figure 5(a)), the streamwise turbulence spectrum, $E_{11}$, decays uniformly at all frequencies. A similar behavior was also found by Gea-Aguilera et al. ${ }^{23}$ for a two-dimensional airfoil, and was due to the reduction of the mean flow velocity in the chordwise direction along the stagnation line. The transverse and spanwise turbulence spectra, $E_{22}$ and $E_{33}$ respectively, show similar trends, with a slight increase $(\sim 2 \mathrm{~dB})$ at low frequencies that tends to zero as the frequency increases.

In the root region (see Figure 5(b)), the distortions are more pronounced and the behaviour more complex. The streamwise turbulence spectrum is amplified at high frequencies, with small variations at low frequencies. The transverse turbulence spectrum is increased at low frequencies and decreased at high frequencies, whereas the spanwise turbulence spectrum follows the opposite trend. The behaviour of the distorted $E_{22}$ in the root region, which is the dominant turbulence spectrum on the leading edge noise of a flat plate, ${ }^{21}$ is similar to that reported by Gea-Aguilera et al..$^{23}$ and Hainaut et al. ${ }^{34}$ in the vicinity of two-dimensional thick airfoils.

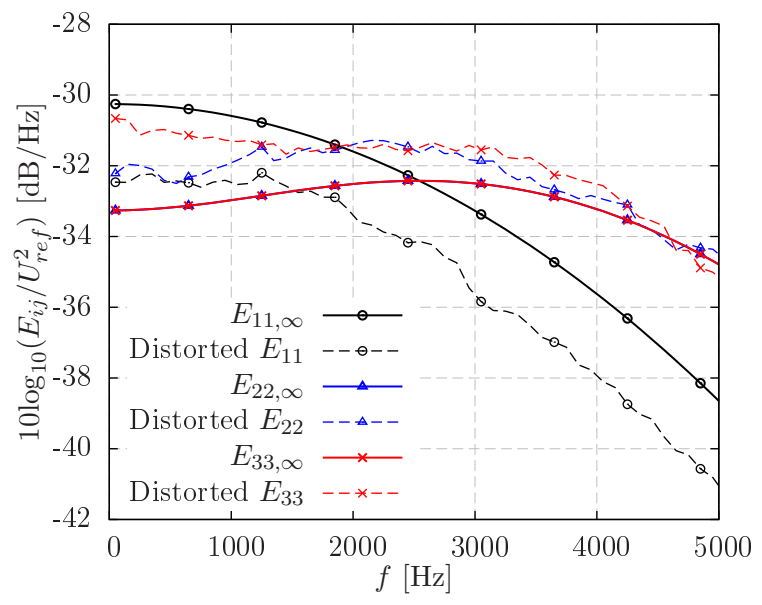

(a) Peak region.

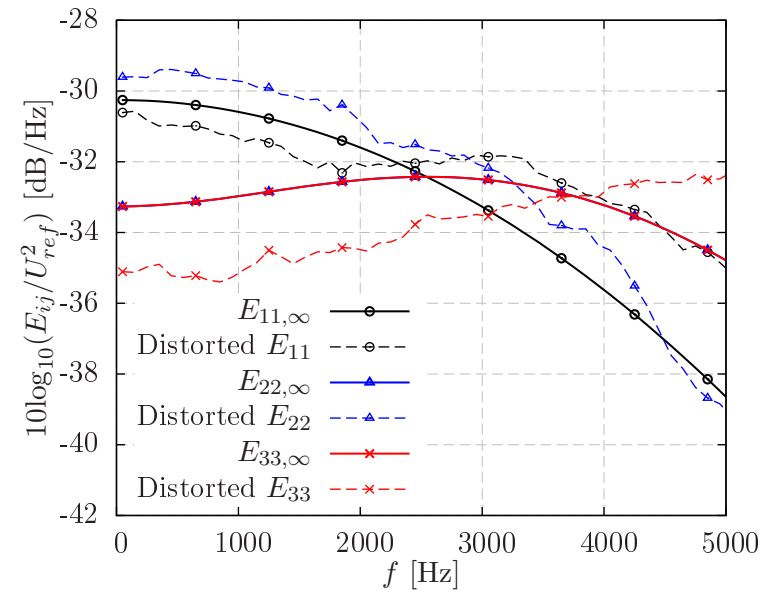

(b) Root region.

Figure 5. Distorted one-dimensional spectra of isotropic turbulence (with $l_{x}=l_{y}=l_{z}=0.008 \mathrm{~m}$ ) calculated at $0.005 c_{0}$ upstream of the peak and root of the wavy leading edge on the symmetry plane, $y / c_{0}=0$.

There are significant differences between the distortion of the turbulence spectra at the peak and root regions of a wavy leading edge airfoil. Figure 6 shows the distortions of the turbulence spectra for various degrees of anisotropy in the oncoming turbulent flow. At the peak region, anisotropic turbulence spectra, $E_{11}, E_{22}$ and $E_{33}$, follow similar trends to the isotropic turbulence case. A possible explanation for this is that the airfoil peaks interact with a clean turbulent flow before it is significantly effected by the strong spanwise pressure gradients towards the root regions. ${ }^{12}$ However, the spanwise length scale, $l_{z}$, seems to play a role in the distortion of the turbulence spectra in the root region. Particularly, the transverse turbulence spectrum is increased as the spanwise length scale increases $\left(l_{x}=l_{y}=l_{z} / 2=0.008 \mathrm{~m}\right)$, whereas the streamwise and spanwise turbulence spectra are decreased. The opposite trend occurs when the spanwise length scale is decreased $\left(l_{x}=l_{y}=2 l_{z}=0.008 \mathrm{~m}\right)$ as shown in Figure 6 . Variations in the chordwise length scale (cases with $l_{x} / 2=l_{y}=l_{z}=0.008 \mathrm{~m}$ and $2 l_{x}=l_{y}=l_{z}=0.008 \mathrm{~m}$ ) do not seem to alter the conclusions discussed for isotropic turbulence. This result suggests that moderate variations in the spanwise length scale may have a larger effect on the leading edge noise than variations in the chordwise length scale. 


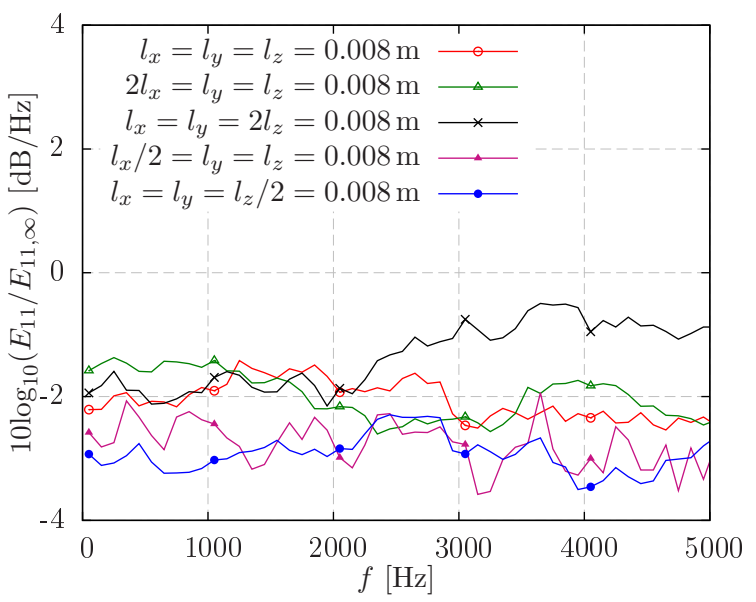

(a) Distorted $E_{11}$ in the peak region.

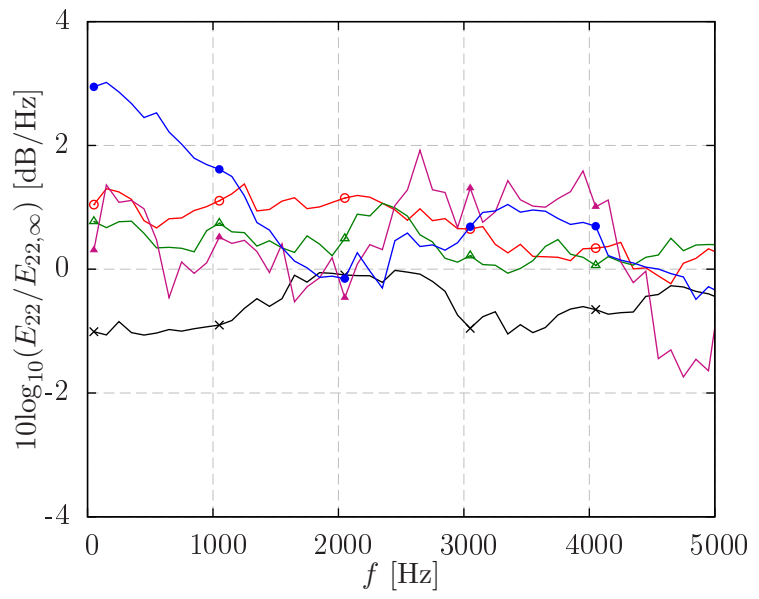

(c) Distorted $E_{22}$ in the peak region.

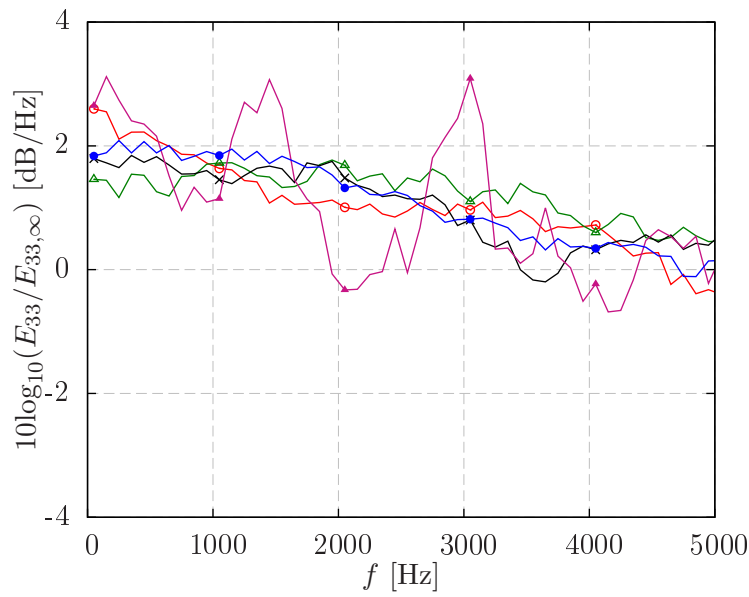

(e) Distorted $E_{33}$ in the peak region.

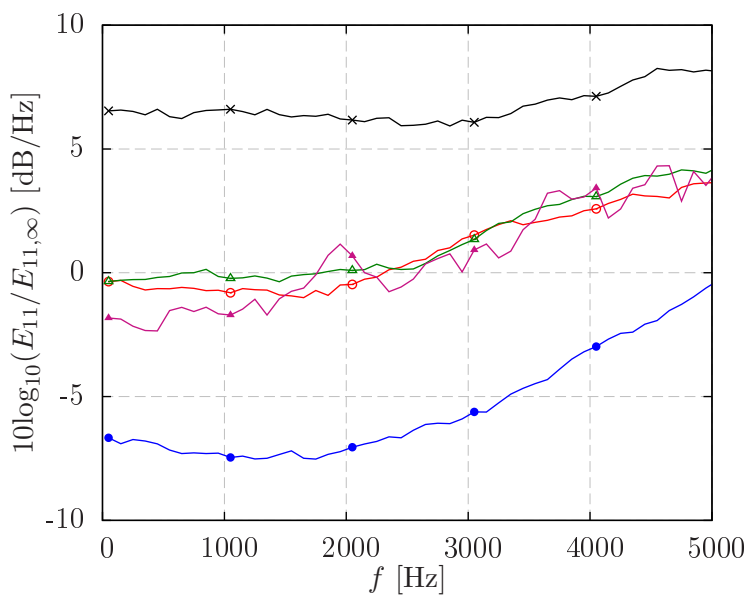

(b) Distorted $E_{11}$ in the root region.

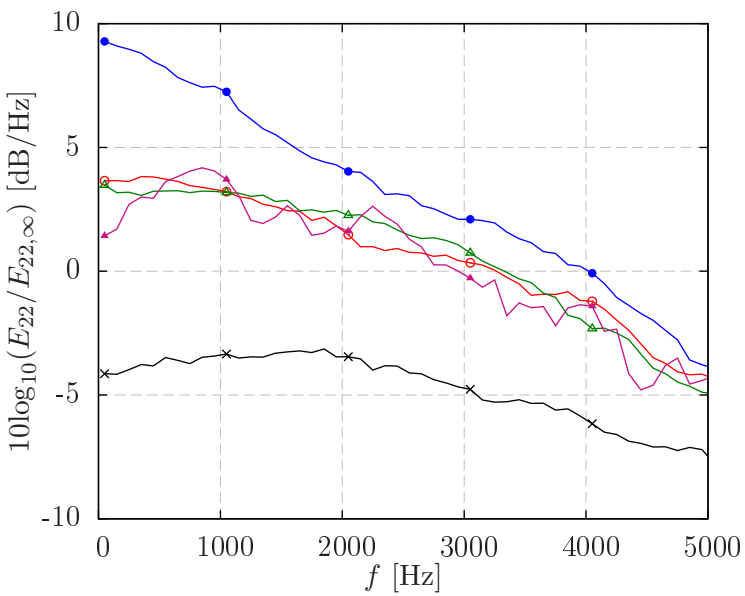

(d) Distorted $E_{22}$ in the root region.

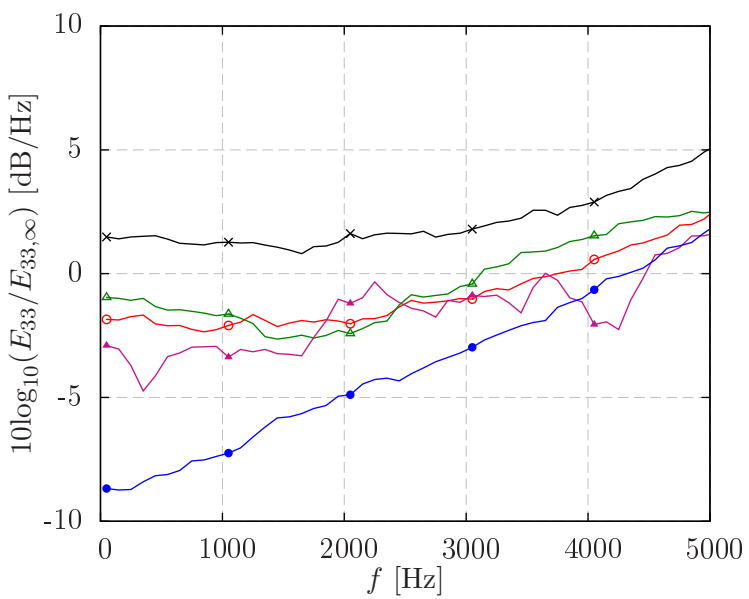

(f) Distorted $E_{33}$ in the root region.

Figure 6. Distorted one-dimensional spectra for anisotropic turbulence calculated at $0.5 \%$ of $c_{0}$ upstream of the peak and root of the wavy leading edge.

Another relevant feature of anisotropic turbulent flows is related to the distribution of the turbulent kinetic energy, $K$, over the different fluctuating velocity components (see Table 1). As a consequence, two turbulent flows with the same turbulent kinetic energy can produce different noise levels depending 
on the turbulence length scales involved. Figure 7 shows a comparison of anisotropic turbulent flows with variations in the chordwise and spanwise length scales but similar turbulent kinetic energy $\left(K=16.07 \mathrm{~m}^{2} / \mathrm{s}^{2}\right.$ and $K=17.01 \mathrm{~m}^{2} / \mathrm{s}^{2}$ in Table 1). Stretching the turbulent structures in the spanwise direction increases the amplitude of the turbulence spectra at high frequencies, whereas an increase in the chordwise length scale is associated with an increase at low frequencies.

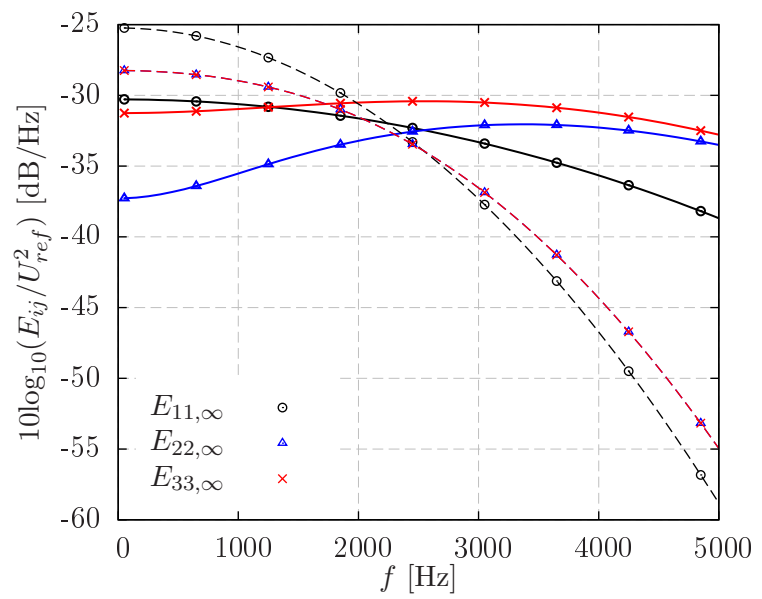

(a) Cases with $K=16.07 \mathrm{~m}^{2} / \mathrm{s}^{2}$. Solid lines correspond to $l_{x}=l_{y}=l_{z} / 2=0.008 \mathrm{~m}$ and dashed lines correspond to $l_{x} / 2=l_{y}=l_{z}=0.008 \mathrm{~m}$.

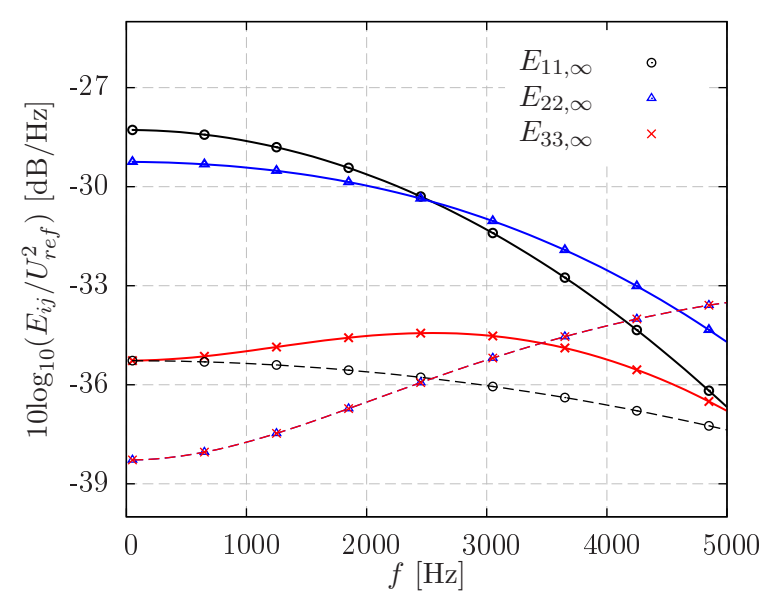

(b) Cases with $K=17.01 \mathrm{~m}^{2} / \mathrm{s}^{2}$. Solid lines correspond to $l_{x}=l_{y}=2 l_{z}=0.008 \mathrm{~m}$ and dashed lines correspond to $2 l_{x}=$ $l_{y}=l_{z}=0.008 \mathrm{~m}$.

Figure 7. Comparison of one-dimensional spectra for anisotropic turbulence with constant turbulent kinetic energy (from Table 1) and variations in the turbulence length scales.

\section{VI.B. Unsteady Pressure on the Airfoil Surface}

The noise perceived by an observer in the far-field is a consequence of the near-field pressure fluctuations that are subsequently radiated to the far-field. This section examines the unsteady pressure field on the airfoil surface from CAA simulations using isotropic and anisotropic turbulence.

Figure 8(a) shows contours of root-mean-square fluctuating pressure, $p_{r m s}^{\prime}$, on the airfoil surface for isotropic turbulence. As highlighted in previous numerical works for flat plates ${ }^{8}$ or using parallel gusts, ${ }^{22}$ the noise sources are located in the peak and root regions, with the noise sources in the root region being dominant. The hill between peaks and roots does not contribute significantly to the radiated noise. ${ }^{8,22}$

Anisotropic turbulence may introduce variations in the relative strengths of the noise sources along the leading edge. For instance, Figure $8(\mathrm{~b})$ shows $\Delta p_{r m s}^{\prime}$ to compare the unsteady pressure generated by turbulence shrunk in the spanwise direction $\left(l_{x}=l_{y}=2 l_{z}=0.008 \mathrm{~m}\right)$ against turbulence shrunk in the chordwise direction $\left(2 l_{x}=l_{y}=l_{z}=0.008 \mathrm{~m}\right)$. For moderate variations of the turbulence length scales, the variations in $\Delta p_{r m s}^{\prime}$ are small and the location of the noise sources remains unchanged. However, it is possible to conclude that the noise from the stagnation region in the peaks and roots is increased for cases with $l_{x}>l_{z}$. Additionally, the noise sources on the sides of the peak region are expected to become increasingly important when $l_{z}>l_{x}$.

Contours of root-mean-square fluctuating pressure are useful to locate the noise sources, but do not provide any information about their frequency content. For isotropic turbulence, Figure 9 shows the spectral content of the unsteady pressure response on the airfoil surface along the chord in terms of SPL. It can be seen that the noise is mainly radiated from locations close to the airfoil leading edge $\left(x / c_{0}<0.2\right)$ and low reduced frequencies $\left(f c_{0} / U_{\infty}<4\right)$, i.e., the maximum in $p_{r m s}^{\prime}$ contours in Figure 8 (a) is primarily caused by low frequencies. 


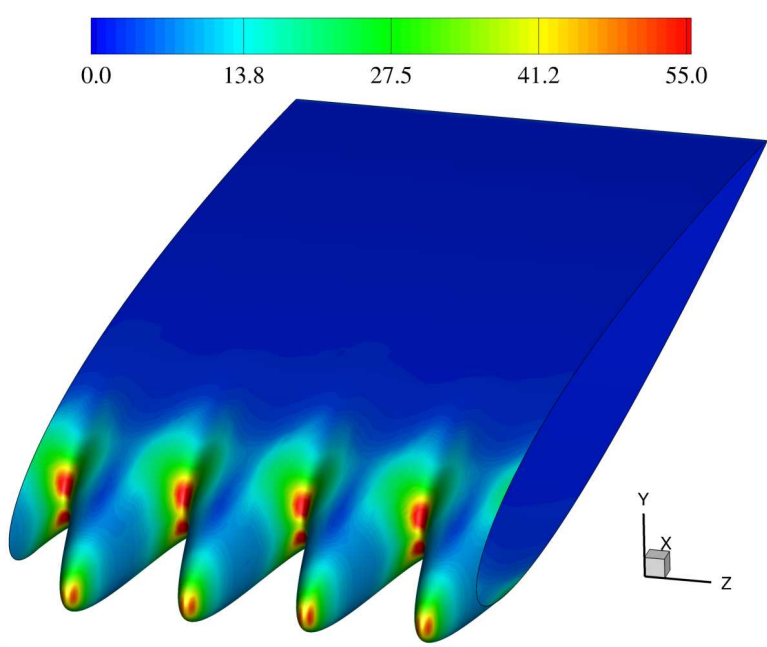

(a) Contours of $p_{r m s}^{\prime}(\mathrm{Pa})$ for isotropic turbulence.

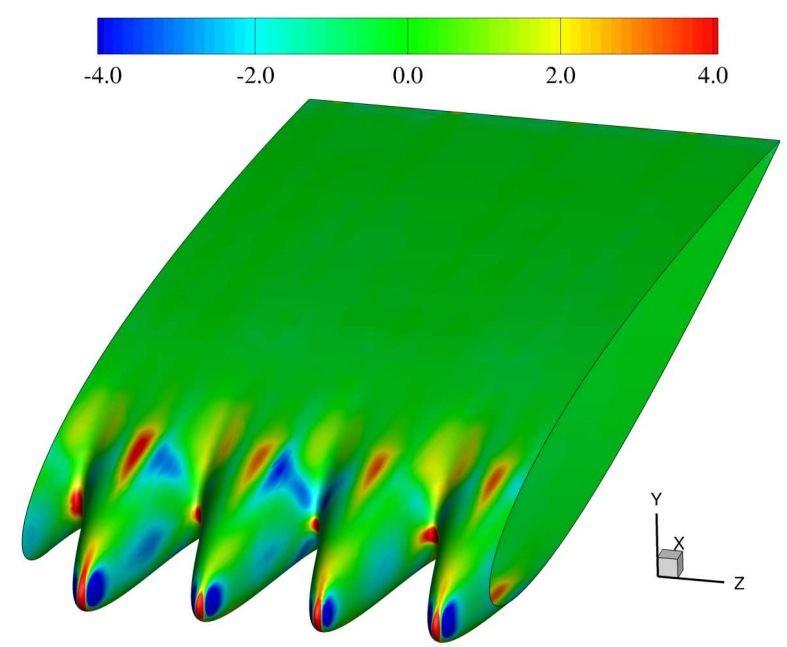

(b) Contours of $\Delta p_{r m s}^{\prime}=\left.p_{r m s}^{\prime}\right|_{l_{x}=l_{y}=2 l_{z}=0.008 \mathrm{~m}-}$

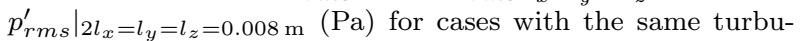
lent kinetic energy but different turbulence length scales.

Figure 8. Unsteady pressure distribution on the surface of a thick airfoil with a wavy leading edge.

For the peak cross-section, there appears to be a secondary noise source at the location of the root (see Figure $9\left(\right.$ a) at $x / c_{0}=0.15$ ), which is a consequence of the high strength of the noise source in the root region. For the hill cross-section, the noise is not radiated from the leading edge, but from a location that corresponds to the root of the wavy leading edge $\left(x / c_{0}=0.15\right)$. Nevertheless, the noise levels radiated from for the hill cross-section are at least $10 \mathrm{~dB}$ lower at all frequencies than for the peak and root cross-sections. This means that the contribution of the hill cross-section of a thick airfoil to the overall noise level is small, as previously found for flat plates.

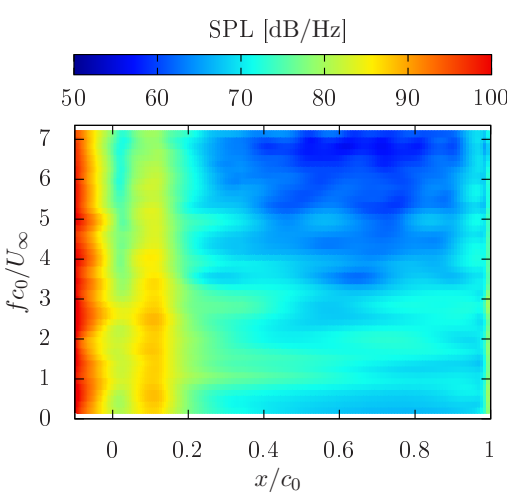

(a) Peak cross-section.

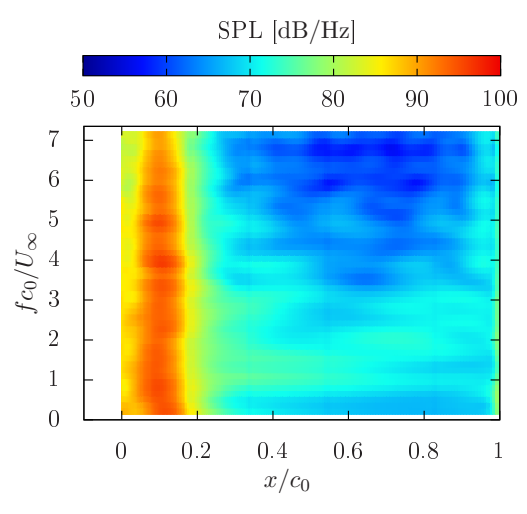

(b) Hill cross-section.

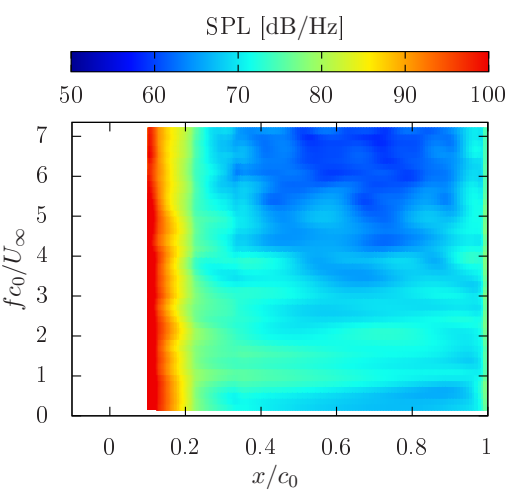

(c) Root cross-section.

Figure 9. SPL spectra for various cross-sections of the wavy leading edge airfoil interacting with isotropic turbulence.

Figures 10 and 11 show $\Delta$ SPL on the airfoil surface at various cross-sections for turbulent flows with the same turbulent kinetic energy but different turbulence length scales. Particularly, Figure 10 shows $\Delta$ SPL to compare the noise levels generated by turbulence stretched in the spanwise direction $\left(l_{x}=l_{y}=l_{z} / 2=\right.$ $0.008 \mathrm{~m})$ against turbulence stretched in the chordwise direction $\left(l_{x} / 2=l_{y}=l_{z}=0.008 \mathrm{~m}\right)$. All the three cross-sections show an increase in noise levels of up to $25 \mathrm{~dB}$ at high frequencies and a decrease at low frequencies of approximately $15 \mathrm{~dB}$. This implies that stretching the spanwise turbulence length scale above a certain limit reduces the effectiveness of the leading edge serrations more than stretching the chordwise length scale. It can also be seen that the variations in the noise levels in Figure 10 follow the same trends as discussed in Figure 7(a) for the turbulence spectra. Therefore, the anisotropy of a turbulent flow affects the frequency content of the noise sources along the leading edge undulations. 
Although small variations in $\Delta p_{r m s}^{\prime}$ were shown in Figure 8(b) for moderately anisotropic turbulence, the frequency content of the noise sources on the airfoil surface is significantly altered, as shown in Figure 11. Consequently, the noise spectra in the far-field are expected to be effected.

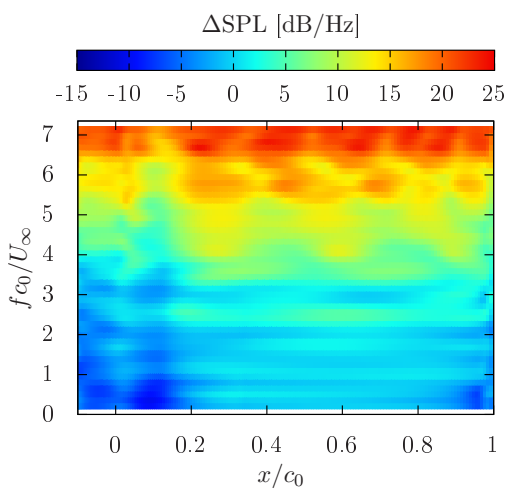

(a) Peak cross-section

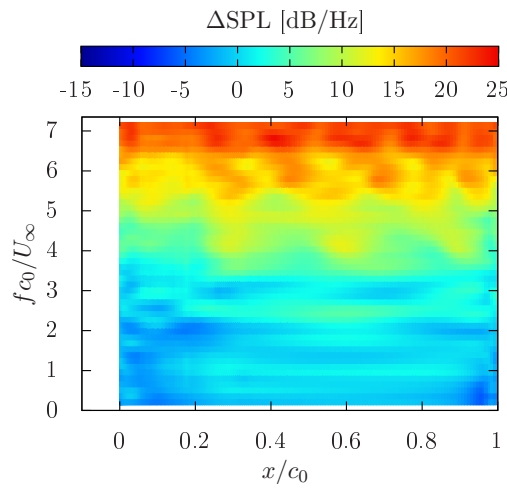

(b) Hill cross-section.

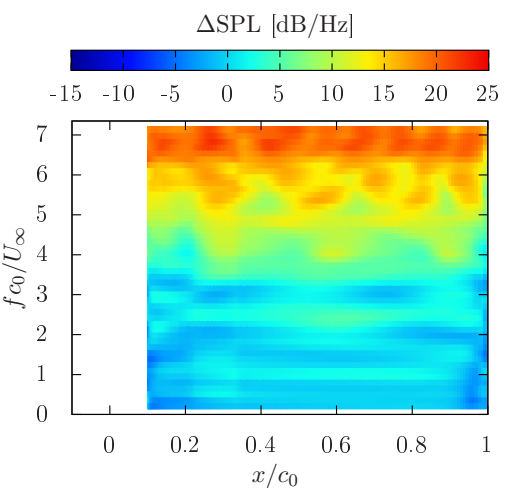

(c) Root cross-section.

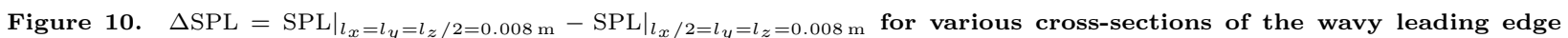
airfoil interacting with anisotropic turbulence.

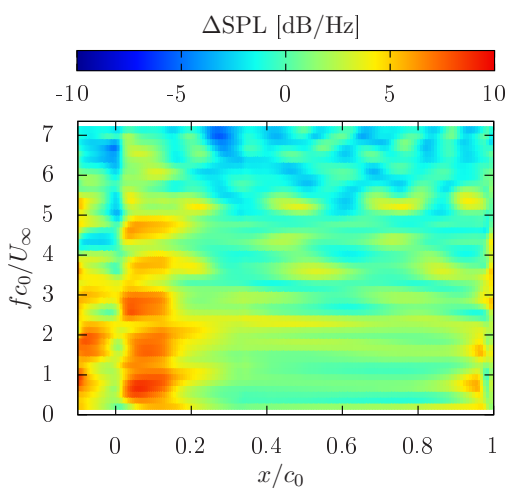

(a) Peak cross-section.

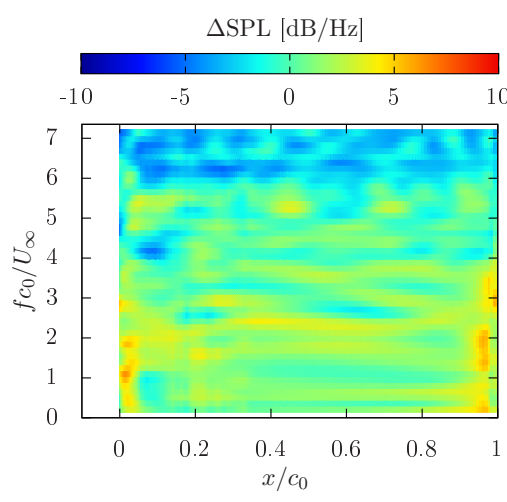

(b) Hill cross-section.

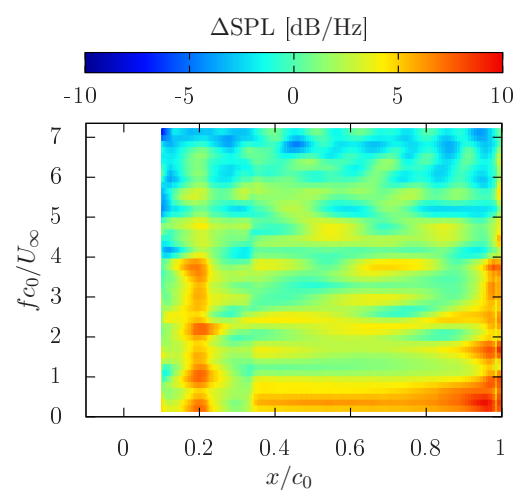

(c) Root cross-section.

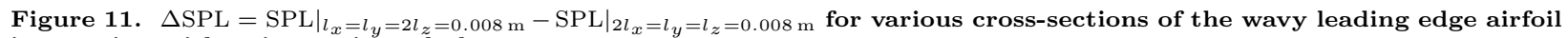
interacting with anisotropic turbulence.

\section{VI.C. Coherence between Distorted Velocity Spectra and Unsteady Pressure}

To study the relationship between the distorted turbulent structures and the unsteady pressure response on the airfoil surface, the magnitude-squared coherence is defined as,

$$
\gamma_{u_{i} p}^{2}(f)=\frac{\left|S_{u_{i} p}(f)\right|^{2}}{S_{u_{i} u_{i}}(f) S_{p p}(f)},
$$

where $S_{u_{i} u_{i}}$ is the spectral density of the fluctuating velocity $\left(u_{i}^{\prime}\right)$ in the vicinity of the leading edge, $S_{p p}$ is the spectral density of the fluctuating pressure $\left(p^{\prime}\right)$ on the airfoil surface, and $S_{u_{i} p}$ is the cross-spectral density between $u_{i}^{\prime}$ and $p^{\prime}$. The aim is to identify the fluctuating velocity component that contributes the most to the strength of the noise sources on the airfoil surface.

Six monitor points (A, B, C, D, E and F) were placed on the airfoil surface to extract the time series of the pressure fluctuations in the peak and root regions, where the noise sources are located (see Figure 12(a)). Figures 12(b), 12(c) and 12(d) show the coherence between distorted velocity fluctuations collected $0.005 c_{0}$ upstream of the peak and root cross-sections along the stagnation line and pressure fluctuations on the surface of a NACA 0012 airfoil at various locations. To simplify the discussion, this study is restricted to isotropic turbulence in this section $\left(l_{x}=l_{y}=l_{z}=0.008 \mathrm{~m}\right)$. 
In the peak region, the noise source is generated by contributions from all the three fluctuating velocity components. At the stagnation point (location A), velocity fluctuations in the streamwise direction are dominant $\left(\gamma_{u_{x} p}^{2}\right.$ approximately 0.8). Moving in the spanwise direction in the peak region (location B) shows that the velocity fluctuations in the spanwise direction are dominant $\left(\gamma_{u_{z} p}^{2}>0.9\right)$. Finally, the velocity fluctuations in the transverse direction are dominant at location $\mathrm{C}\left(\gamma_{u_{y} p}^{2}\right.$ approximately 0.7$)$.

In the root region, the noise source close to the maximum $p_{r m s}^{\prime}$ (location $\mathrm{F}$ ) is mainly generated by the velocity fluctuations in the transverse direction $\left(\gamma_{u_{y} p}^{2} \approx 0.5\right)$, as occurs for flat plates and airfoils with straight leading edge. Therefore, velocity fluctuations in the transverse direction (normal to the chord) are the main contributor to the noise from airfoils with wavy leading edge interacting with isotropic turbulence. This result partially justifies the approach of Clair et al. ${ }^{22}$ which only modelled parallel gusts with transverse disturbances to compute the noise from a wavy leading edge airfoil in CAA simulations. On the symmetry plane (locations D and E), the noise source is weak, and the main contribution is the fluctuating velocity component in the streamwise direction, at least at low frequencies.

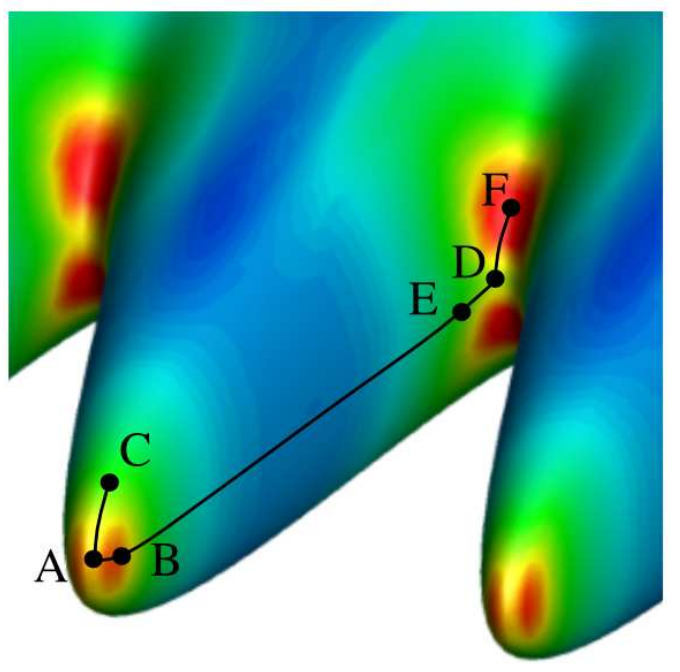

(a) Monitor points on the airfoil surface to extract the pressure fluctuations.

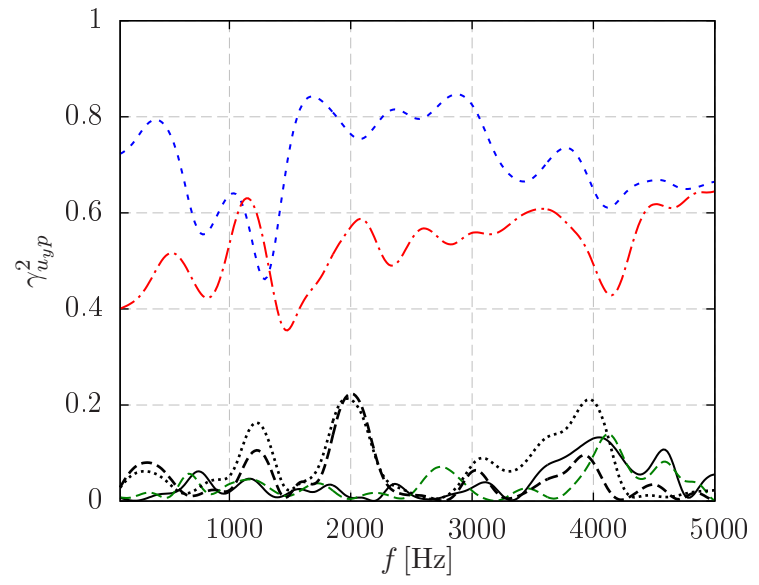

(c) Coherence $\gamma_{u_{y} p}^{2}$.

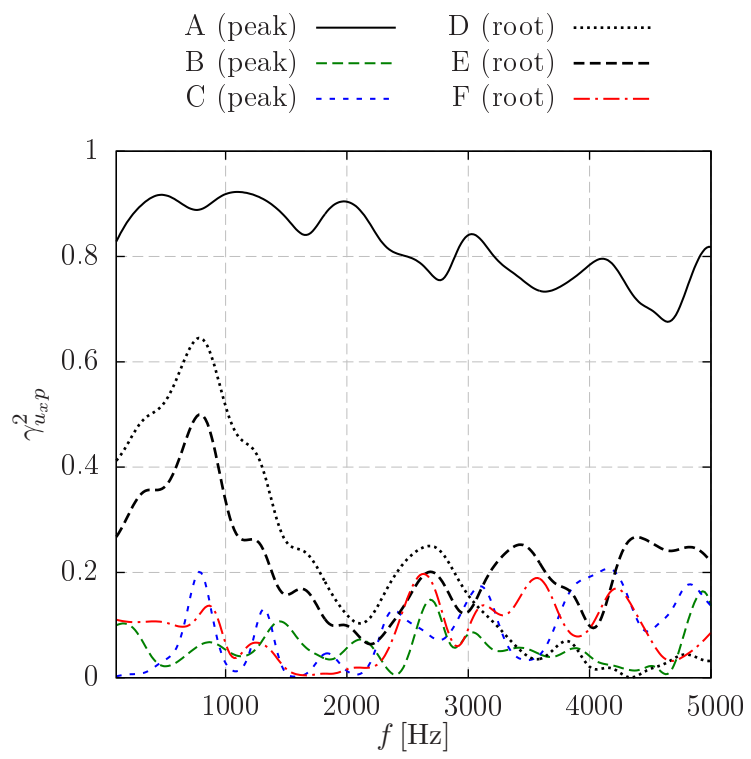

(b) Coherence $\gamma_{u_{x} p}^{2}$.

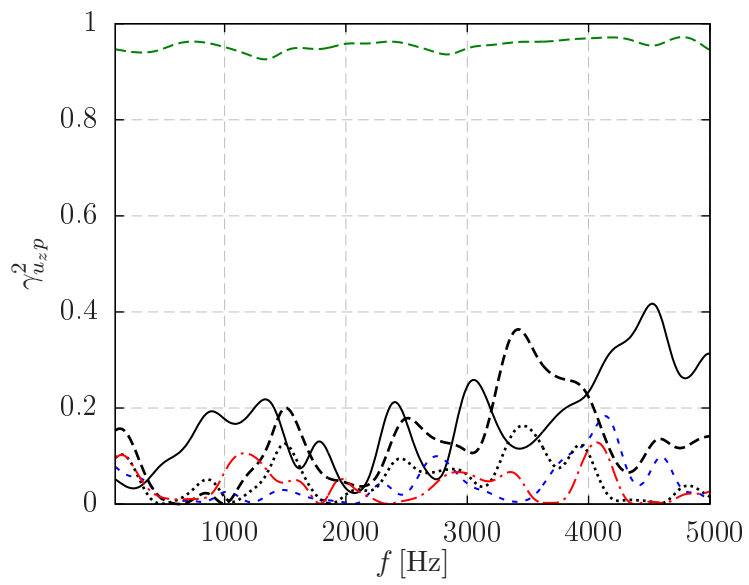

(d) Coherence $\gamma_{u_{z} p}^{2}$.

Figure 12. Coherence between distorted velocity fluctuations in the vicinity of the wavy leading edge and pressure fluctuations on the airfoil surface at various locations. 


\section{VI.D. Correlation between Noise Sources along the Span}

The effectiveness of airfoils with wavy leading edge is based on the decorrelation between noise sources in the chordwise and spanwise directions. However, such a decorrelation effect can be masked if the amplitude of the undulations, $2 h_{w}$, and their wavelength, $\lambda_{w}$, are not optimized based on the spanwise and chordwise length scales of the turbulent structures. To study this phenomenon, the auto-correlation of the pressure fluctuations along the span is calculated as,

$$
R_{p p}(z)=\frac{\left\langle p^{\prime}\left(\mathbf{x}_{0}+z, t\right) p^{\prime}\left(\mathbf{x}_{0}, t\right)\right\rangle}{\left\langle p^{\prime}\left(\mathbf{x}_{0}, t\right) p^{\prime}\left(\mathbf{x}_{0}, t\right)\right\rangle}
$$

where $\mathbf{x}_{0}$ is the reference point, which is taken at the center of the root region.

Figure 13 shows the auto-correlation of the noise sources along the span. Note that two different $y / t_{A}$ planes have been considered since the maximum $p_{r m s}^{\prime}$ occurs at different $y$-locations for a thick airfoil. It can be seen that for a fixed wavelength of the undulations, $\lambda_{w}=0.016 \mathrm{~m}$, the auto-correlation between two consecutive roots $\left(z / \lambda_{w}=0\right.$ and $\left.z / \lambda_{w}=1\right)$ is increased as the spanwise length scale, $l_{z}$, increases. This result highlights the importance of optimizing $l_{z} / \lambda_{w}$. For cases with $l_{z} / \lambda_{w} \leq 0.5$ noise sources in the root region along the span are uncorrelated, and therefore, wavy leading edge airfoils are expected to reduce far-field noise levels. This result is consistent with the criterion proposed by Chaitanya et al. ${ }^{6}$ for isotropic turbulence. However, cases with $l_{z} / \lambda_{w} \geq 0.75$, present an increasing correlation of the noise sources in the spanwise direction, which could potentially reduce the effectiveness of the serrations.

Moderate variations in the chordwise length scale, $l_{x}$, have no significant influence on the correlation between noise sources along the span. This is because the majority of cases investigated in this work present $l_{x} /\left(2 h_{w}\right)<1$, and therefore, the amplitude of the serrations is larger than the chordwise length scale of the turbulent structures. However, an increased correlation between noise sources at the peak and root can be found in $R_{p p}$ for the case with $l_{x} /\left(2 h_{w}\right)>1$ in the $y / t_{A}=0$ plane (Figure 13(a)). This result indicates that correlations between noise sources in the peak and root region may occur for sufficiently large turbulent structures in the streamwise direction. Nevertheless, the correlation between peak and root noise sources is expected to have a minor contribution to the far-field noise than the correlation between two consecutive roots, where the maximum $p_{r m s}^{\prime}$ on the wavy leading edge is located.

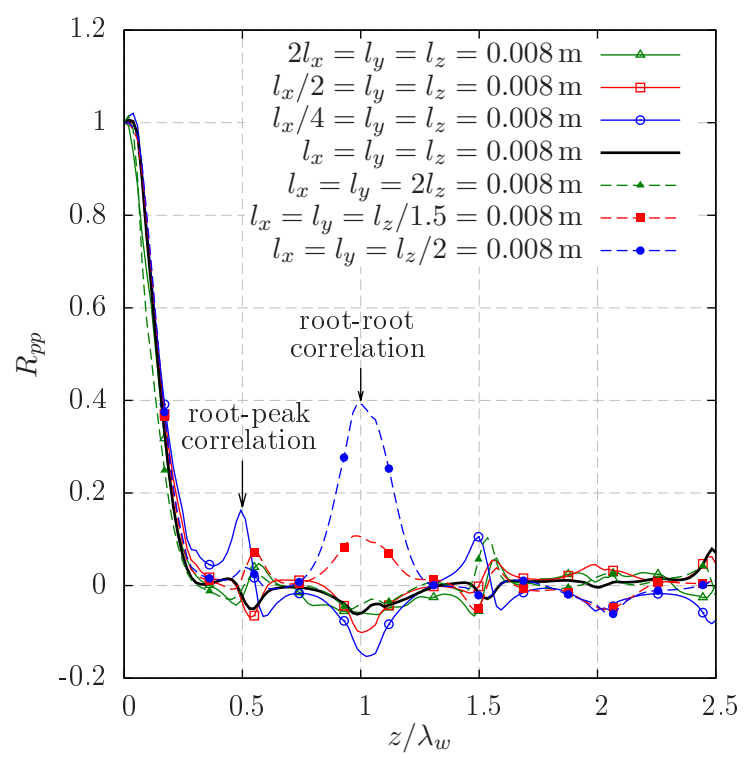

(a) Plane of the maximum $p_{r m s}^{\prime}$ in the peak region $\left(y / t_{A}=0\right)$.

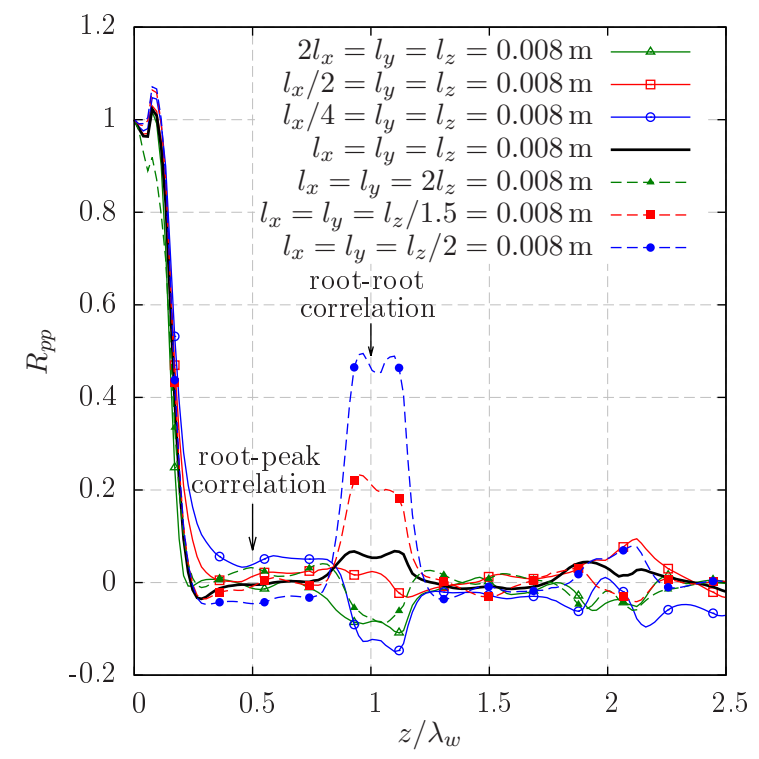

(b) Plane close to the maximum $p_{r m s}^{\prime}$ in the root region $\left(y / t_{A}=0.25\right)$.

Figure 13. Correlation of pressure fluctuations along the wavy leading edge in the spanwise direction. 


\section{Conclusions}

A synthetic turbulence method that is able to generate three-dimensional homogeneous anisotropic turbulence has been developed, implemented and validated in an LEE solver. The resulting turbulent flow is divergence-free, as required in CAA simulations to avoid spurious noise sources in the CAA domain. The method has been used to study turbulence-airfoil interaction noise from a NACA 0012 airfoil with wavy leading edge interacting with moderately anisotropic turbulence. The aim is to improve current understanding of the noise generation mechanism of airfoils with wavy leading edges, and to optimize their geometry based on the turbulence length scales. For instance, this would potentially help to design low-noise OGVs with serrations based on the anisotropy in the fan wakes. The main findings of this paper are summarized as follows:

- Distortions of the turbulence spectra due to mean flow velocity gradients are more pronounced in the root region than in the peak region. Variations in the spanwise length scale, $l_{z}$, seem to affect the amount of distortion in the turbulence spectra more than the other length scales.

- In anisotropic flows, two turbulent flows with approximately the same turbulent kinetic energy produce noise sources along the leading edge with a different spectral content depending on the turbulence length scales. This highlights the importance of characterizing the length scales in a turbulent flow to optimize wavy leading edge design.

- The locations of the noise sources in the peak and root regions are not significantly effected by the interaction of moderately anisotropic turbulence with wavy leading edge airfoils.

- The frequency content of the noise sources along the wavy leading edge is characterized by the anisotropic turbulence spectra. Stretching the turbulent structures in the spanwise direction increases the amplitude of the turbulence spectra at high frequencies, whereas an increase in the chordwise length scale is mainly associated with an increase at low frequencies.

- The examination of $\Delta p_{r m s}^{\prime}$ between two flows with the same turbulent kinetic energy indicates that stretching the spanwise length scale, $l_{z}$, has a bigger impact in reducing the effectiveness of the leading edge serrations than stretching the chordwise length scale, $l_{x}$.

- The magnitude-squared coherence between velocity and pressure fluctuations indicates that the transverse component of the fluctuating velocity is dominant in the noise sources located in the root region, as occurs for flat plates and airfoils with straight leading edge. However, all the three components of the fluctuating velocity affect the noise sources located in the peak region.

- The maximum decorrelation between noise sources is achieved by ensuring that $l_{z} / \lambda_{w} \leq 0.5$ (for low root-to-root correlation) and $l_{x} /\left(2 h_{w}\right)<1$ (for low root-to-peak correlation). These parameters can be retained to design low-noise airfoils with wavy leading edge interacting with anisotropic turbulence. Among them, minimizing the correlation between two consecutive noise sources in the root regions seems more relevant, at least for the cases examined in this work.

\section{References} (2011).

${ }^{1}$ European Commission, Report of the High Level Group on Aviation, Flightpath 2050 Europe's Vision for Aviation,

${ }^{2}$ Astley, R. J., Agarwal, A., Holland, K. R., Joseph, P. F., Self, R. H., Smith, M. G., Sugimoto, R., Tester, B. J., "Predicting and Reducing Aircraft Noise," 14th International Congress on Sound and Vibration, Australia, 2007.

${ }^{3}$ Ganz, U. W., Joppa, P. D., Patten, T. J., Scharpf, D. F, "Boeing 18-Inch Fan Rig Broadband Noise Test," NASA Contractor Report, CR-1998-208704, 1998.

${ }^{4}$ Podboy, G. G., Krupar, M. J., Helland, S. M., Hughes, C. E., "Steady and Unsteady Flow Field Measurements within a NASA 22-inch Fan Model," 40th Aerospace Sciences Meeting and Exhibit, No. AIAA 2002-1033, 2002; and NASA Technical Memorandum, TM-2003-212329, 2003.

${ }^{5}$ Hersh, A. S., Soderman, P. T., Hayden, R. E., "Investigation of Acoustic Effects of Leading-Edge Serrations on Airfoils," Journal of Aircraft, Vol. 11, No. 4, pp. 197-202, 1974.

${ }^{6}$ Chaitanya, P., Narayanan, S., Joseph, P., Vanderwel, C., Turner, J., Kim, J. W., Ganapathisubramani, B., "Broadband Noise Reduction through Leading Edge Serrations on Realistic Aerofoils," 21st AIAA/CEAS Aeroacoustics Conference, No. AIAA 2015-2202, 2015. 
${ }^{7}$ Chaitanya, P., Joseph, P., Narayanan, S., Vanderwel, C., Turner, J., Kim, J. W., Ganapathisubramani, B., "Performance and Mechanism of Sinusoidal Leading Edge Serrations for the Reduction of Turbulence-Aerofoil Interaction Noise," Journal of Fluid Mechanics, Vol. 818, pp. 435-464, 2017.

${ }^{8}$ Kim, J. W., Haeri, S., Joseph, P., "On the Reduction of Aerofoil-Turbulence Interaction Noise Associated with Wavy Leading Edges," Journal of Fluid Mechanics, Vol. 792, pp. 526-552, 2016.

${ }^{9}$ Soderman, P. T., "Aerodynamic Effects of Leading-Edge Serrations on a Two-Dimensional Airfoil," NASA Technical Memorandum, TM X-2643, 1972.

${ }^{10}$ Miklosovic, D. S., Murray, M. M., Howle, L. E., Fish, F. E., "Leading-Edge Tubercles Delay Stall on Humpback Whale (Megaptera Novaeangliae) Flippers," Physics of Fluids, Vol. 16, No. 5, pp. 39-42, 2004.

${ }^{11}$ van Nierop, E. A., Alben, S., Brenner, M. P., "How Bumps on Whale Flippers Delay Stall: An Aerodynamic Model," Physical Review Letters, Vol. 100, No. 5, pp. 054502, 2008.

${ }^{12}$ Skillen, A., Revell, A., Pinelli, A., Piomelli, U., Favier, J., "Flow over a Wing with Leading-Edge Undulations," AIAA Journal, Vol. 53, No. 2, pp. 464-472, 2015.

${ }^{13}$ Bachmann, T., Klän, S., Baumgartner, W., Klaas, M., Schröder, W., Wagner, H., "Morphometric Characterisation of Wing Feathers of the Barn Owl Tyto Alba Pratincola and the Pigeon Columba Livia," Frontiers in Zoology, Vol. 4, No. 23, 2007.

${ }^{14}$ Lau, A. S. H., Haeri, S., Kim, J. W., "The Effect of Wavy Leading Edges on Aerofoil-Gust Interaction Noise," Journal of Sound and Vibration, Vol. 332, No. 24, pp. 6234-6253, 2013.

${ }^{15}$ Mathews, J., Peak, N., "Noise Generation by Turbulence Interacting with an Aerofoil with a Serrated Leading Edge," 21st AIAA/CEAS Aeroacoustics Conference, No. AIAA 2015-2204, 2015.

${ }^{16}$ Narayanan, S., Chaitanya, P., Haeri, S., Joseph, P., Kim, J. W., Polacsek, C., "Airfoil Noise Reductions Through Leading Edge Serrations," Physics of Fluids, Vol. 27, pp. 025109, 2015.

${ }^{17}$ Gea-Aguilera, F., Gill, J., Zhang, X., Chen, X., Nod-Langlois, T., "Leading Edge Noise Predictions Using Anisotropic Synthetic Turbulence," 22nd AIAA/CEAS Aeroacoustics Conference, No. AIAA 2016-2840, 2016.

${ }^{18}$ Chaitanya, P., Narayanan, S., Joseph, P., Kim, J. W., "Leading Edge Serration Geometries for Significantly Enhanced Leading Edge Noise Reductions," 22nd AIAA/CEAS Aeroacoustics Conference, No. AIAA 2016-2736, 2016.

${ }^{19}$ Turner, J., Kim, J. W., Chaitanya, P., Joseph, P., "Towards Understanding Aerofoils with Dual-Frequency Wavy Leading Edges Interacting with Vortical Disturbances," 22nd AIAA/CEAS Aeroacoustics Conference, No. AIAA 2016-2951, 2016.

${ }^{20}$ Roger, M., Schram, C., De Santana, L., "Reduction of Airfoil Turbulence-Impingement Noise by Means of Leading-Edge Serrations and/or Porous Materials," 19th AIAA/CEAS Aeroacoustics Conference, No. AIAA 2013-2108, 2013.

${ }^{21}$ Amiet, R. K., "Acoustic Radiation from an Airfoil in a Turbulent Stream," Journal of Sound and Vibration, Vol. 41, No. 4, pp. 407-420, 1975.

${ }^{22}$ Clair, V., Polacsek, C., Le Garrec, T., Reboul, G., Gruber, M., Joseph, P., "Experimental and Numerical Investigation of Turbulence-Airfoil Noise Reduction using Wavy Edges," AIAA Journal, Vol. 51, No. 11, pp. 2695-2713, 2013.

${ }^{23}$ Gea-Aguilera, F., Zhang, X., Chen, X., Gill, J., Nodé-Langlois, T., "Synthetic Turbulence Methods for Leading Edge Noise Predictions," 21st AIAA/CEAS Aeroacoustics Conference, No. AIAA 2015-2670, 2015.

${ }^{24}$ Gea-Aguilera, F., Gill, J., Zhang, X., Nodé-Langlois, T., "Turbulence-Cascade Interaction Noise Using an Advanced Digital Filter Method," 23rd International Congress on Sound and Vibration, 2016.

${ }^{25}$ Gea-Aguilera, F., Gill, J., Zhang, X., Nodé-Langlois, T., "Fan Wake Modelling for Computational Aeroacoustic Simulations of Turbulence-Cascade Interaction Noise," Greener Aviation, 2016.

${ }^{26}$ Kim, J. W., "Optimised Boundary Compact Finite Difference Schemes for Computational Aeroacoustics," Journal of Computational Physics, Vol. 225, No. 1, pp. 995-1019, 2007.

${ }^{27} \mathrm{Kim}$, J. W., "High-Order Compact Filters with Variable Cut-Off Wavenumber and Stable Boundary Treatment," Journal of Computers and Fluids, Vol. 39, No. 7, pp. 1168-1182, 2010.

${ }^{28} \mathrm{Hu}$, F. Q., Hussaini, M. Y., Manthey, J., "Low-Dissipation and -Dispersion Runge-Kutta Schemes for Computational Acoustics," Journal of Computational Physics, Vol. 124, pp. 177-191, 1996.

${ }^{29}$ Gill, J., Fattah, R., Zhang, X., "Evaluation and Development of Non-Reflective Boundary Conditions for Aeroacoustic Simulations," 21st AIAA/CEAS Aeroacoustics Conference, No. AIAA 2015-2677, 2015.

${ }^{30}$ Thomson, D. J., "Spectrum Estimation and Harmonic Analysis," Proceedings of the Institute of Electrical and Electronics Engineers (IEEE), Vol. 70, No. 9, pp. 10551096, 1982.

${ }^{31}$ Chaitanya, P., Gill, J., Narayanan, S., Joseph, P., Vanderwel, C., Zhang, X., Ganapathisubramani, B., "Aerofoil Geometry

Effects on Turbulence Interaction Noise," 21st AIAA/CEAS Aeroacoustics Conference, No. AIAA 2015-2830, 2015.

${ }^{32}$ Gill, J., Zhang, X., Joseph, P., "Symmetric Airfoil Geometry Effects on Leading Edge Noise," Journal of the Acoustical Society of America, Vol. 134, No. 4, pp. 2669-2680, 2013.

${ }^{33}$ Santana, L. D., Christophe, J., Schram, C., Desmet, W. "A Rapid Distortion Theory Modified Turbulence Spectra for Semi-Analytical Airfoil Noise Prediction," Journal of Sound and Vibration, Vol. 383, pp. 349363, 2016.

${ }^{34}$ Hainaut, T., Gabard, G., Clair, V., "A CAA Study of Turbulence Distortion in Broadband Fan Interaction Noise," 22nd AIAA/CEAS Aeroacoustics Conference, No. AIAA 2016-2839, 2016.

${ }^{35}$ Kim, D., Lee, G.-S., Cheong, C., "Inflow Broadband Noise from an Isolated Symmetric Airfoil Interacting with Incident Turbulence," Journal of Fluids and Structures, Vol. 55, pp. 428450, 2015. 